INTERNATIONAL JOURN AL OF RESEARCHES IN BIOSCIENCES, AGRICULTURE AND TECHNOLOGY (c) VISHWASHANTI MULTIPURPOSE SOCIETY (G lobal Peace Multipurpose Socie ty) R. No. MH-659/13 (N) www.vmsindia.org

\title{
HISTOLOGICAL STUDIES OF INTERSTITIAL CELLS IN THE OVARIES OF BATS HIPPOSIDEROS SPEORIS AND ROUSETTUS LESCHENAULTI
}

\author{
Nitin P. Meshram ${ }^{1}$ and Kishor S. Janbandhu ${ }^{2}$ \\ ${ }^{1 S}$. K. Porwal College, Kamptee, Nagpur [India] \\ ${ }^{2}$ Govt. Institute of Science, Nagpur [India] \\ mnitin_p@yahoo.co.in
}

\begin{abstract}
:
Bats are dominating flying mammals all over the world showing various adaptive specialization. Chiroptera shows great variations in the reproductive stage, even the closely allied species exhibit different breeding habits. It is important to study the reproductive organ that is ovary in respect with the interstitial cells. Because the interstitial cells with the help of LH of pituitary produce a hormone androgen. That means interstitial tissues are developed as a gland having steroidogenic activities. In the immature ovary of Hipposideros speoris, interstitial cells are not distinguished from the stromal cells while It is clearly distinguished from stromal cells in the immature ovary of Rousettus leschenaulti. In immature ovary of Rousettus leschenaulti numerous interstitial cells observed in the stromal area. In the inactive Hipposideros speoris ovary, bundles of interstitial cells observe d $60 \pm 5$. In the inactive ovary of Rousettus leschenaulti interstitial cell bundles obse rved $41 \pm 5$. Number of interstitial cells in each bundle observed in inactive ovary of Hipposideros speoris are 5 to 10 and in Rousettus leschenaulti, it is obse rved 5 to 7 . In the active ovary of Hipposideros speoris, the bundles of interstitial cells observed $80 \pm 5$ and in Rousettus leschenaulti it is observed $47 \pm 5$. Number of interstitial cells in each bundle observed in Hipposideros speoris is 7 to 30. While in Rousettus leschenaulti observed 7 to 20. Pregnant ovary of Rousettus leschenaulti shows introverted carpus luteum, atretic follicles, destructed follicles and interstitial cells. So it is concluded that the interstitial cells in the active ovary is more steroidogenic than inactive ovary.
\end{abstract}

Keyw ords: Hipposideros speoris, Rousettus leschenaulti, interstitial cells

\section{Introduction}

India has a rich chiropteran fauna. 17 families 174 genera and 913 species of bats reported by Koopman (1994). At present 1116 recognized species of bats worldwide Simmons SB (2005) out of the 17 families, 7 families incorporating over of 100 species are reported in India.

A literature survey reveals that meager information is available on the interstitial cells in the ovary of Indian Bats. Guraya (1901), Singh and Krishna (1994), Abhilasha and Krishna (1996) Megaderma lyra lyra breeds end week of November and delivering in $1^{\text {st }}$ to $3^{\text {rd }}$ week of April. Hipposide ros breeds last week of December to $2^{\text {nd }}$ week of March, deliveries in May to mid June.

The present work includes observation on the interstitial cells in ovary of two species of Indian bats megaderma lyra lyra and Hipposideros speoris.

\section{Material and Methods}

Female specimens of megaderma lyra lyra and Hipposideros speoris used for present study collected from the Ballarshah and Kandri mines, near Nagpur in India. The bats were collected from delapidated dark rooms and mines with the help of mist nets and butterfly net, the collection made as per requirement. The animals killed by chloroform and their body weight taken and fixed in $10 \%$ formalin for 24 hours.

\section{HISTOLOGY}

The female reproductive system was dissected out, their ovaries were separated and fixed in $10 \%$ formalin for 24 hours. Tissue washed overnight in running water. The tissue was dehydrated by passing through a graded series of ethyle alcohol, cleared in xylol and embeded in paraffin wax $\left(58^{\circ}-60^{\circ} \mathrm{C}\right)$.

The sections were cut for the thickness of 4 to $5 \mu$ with the help of rotary microtome for routine histological study. The sections were stained with hae matoxyle ne eosin stain.

The photographs were taken with the help of $35 \mathrm{~mm}$ leica camera attached to the microscope and enlarged to the required size. All microscopic observations, measurements were taken with the help of an eyepiece micrometer calibrated to stage microme te $\mathrm{r}$ scale.

\section{HISTORICAL REVIEW}

May J. Guthrie, Katnarine R. Jeffer (1938a) have been studied the ovaries of bats myotis lucifugus lucifugus and $\mathrm{M}$ grisenscence and informed that Interstitial cells very numerous in Newborn bats and they arose from hypertrophied theca internae of retrogressing follicles of granulosa cells. Mossaman et, al (1964) have recently designated that these Interstitial cells of thecal origin in the 
mammalian ovary are of first type of Interstitial cells. The second type of interstitial cells in the ovary of bat are in the form of epithelial cells are irregularly distributed among the first type of inters titial cells.

Brambell FWR (1906) described that the re are several type of glandular tissues in the mammalian ovaries which are severally described as Interstitial cells. The different view regarding their origin have been produced by Brambell to two comprehensive thesis namely epithe lial origin and the connective tis sue origin of the interstitial cells. In many species of mammals the ovarian interstitial tissue consists of cellular clusters and nests Guraya (1967) which are scattered in the cortical stroma. Investigated that ovaries of non pregnant and pregnant grey bat myotis grisensens show two types of Interstitial cells. The first type of cells we re considerably developed and occupy all the ovary except the periphery which contain small follicles they originated by the thecal hypertrophy of atretic large Pre antral and antral follicles and they are apprarently developed to the some extent in the ovaries of pregnant and non pregnant bats. The second type of interstitial cell was in the form of epithelial cells which were irregularly distributed among the first type of interstitial cell.

Sardul S Guraya, Gilbert, S Greenwald (1964) studied various mammalian ovarian interstitial tissues and described that in Rat ovary, the interstitial cells occur as scattered patches of various sizes that are derived from the theca of atretic preantral follicles and antral follicles. Most of Bat ovary, The interstitial cells occupied the total ovarian are a except the cortex and the interstitial cells distributed and scattared in between the follicles and connective tissue area.

\section{Observations}

Interstitial cells in Immature ovary of Hipposideros speoris

The observations in immature ovary of Hipposideros speoris shown that the Interstitial cells cannot be distinguished from the stromal cells. The number of developing primordial follicles seen. Amongst the developing follicles small clusters of cells seen with lightly staining vesicular nuclei in some places, these are forerunner of the interstitial cells. The interstitial cells does not become well diffe rentiated in Hipposideros speoris in the immature animals (fig.1 $\mathrm{a}$ and $1 \mathrm{~b}$ )
Interstiitial cells in mature ovary of Hipposideros speoris

In mature ovary of Hipposideros speoris shows, the larger area of stroma composed of connective tissue cells with elongated nuclei. follicular composition of the ovary shows various different stages of the reproductive cycle development. In inactive ovary only primordial, unilaminor, bilaminar, preovulatory, graffian follicles, antral follicles are found.

Interstitial cells in Inactive ovary of Hipposideros speoris

The inactive ovary of Hipposiderous speoris showed the polyhedral and polygonal shaped scattered interstitial cells found in between various follicles. The interstitial cells are found in small bunches, In the stromal as well as peripheral epithelial area. The connective tissue also seen in the stromal area. The polyhedral interstitial cells having vesicular nuclei. In this stage very few bunches of interstitial cell found distributed in small stromal area because the unilaminar follicles contain large in number and stromal area becomes narrow. In inactive ovary interstitial cells are small about 5 to $7 \mu$. About 5 to 10 cells are consisted in each bunch. There are $60 \pm 5$ such bunches are found in all over the ovarian stroma. (fig 2)

Interstitial cells in active ovary of Hipposideros speoris

The active ovary of Hipposideros speoris noticed that multilaminar follicles, single preovulatory follicle, follicles undergoes artesia. The stroma is compound of polyhedral interstitial cells with rounded nuclei. A few blood capillaries also supplied with interstitial cells. As the primordial follicles are few in number, stromal area becomes larger and number of clusters of interstitial cells are increases. about $80 \pm 5$ bunches of interstitial cells with rounded nuclei are seen distributed among the follicles in the stromal region. Each bunch consisted of about 7 to 30 inters titial cell. Interstitial cells in active ovary is more hypertrophied than inactive ovary are measured about 7 to $10 \mu$. (fig. 3 )

Interstitial cells in Immature ovary of Rousettus leschenaulti

In the immature ovary of Rousettus leschenauti germinal epithelium of ovary is composed of flat cells. Ovarian stroma made up of small ovoid cells and sparsely distributed between the follicles and occupy a major part of the ovary. In the large immature ovary stromal cells are elongated and spindle shaped so that the stroma appears fibrous. The connective 
tissue fibres more abundant and prominant.(Fig4a,4b).

Interstitial cells in Mature Ovary of Rousettus leschenaulti

In the mature ovary of Rousettus leschenaulti the germinal epithelium is composed of cuboidal cells and interstitial cells. The tunica albuginea varies in its thickness in different region of the ovary. The stroma is compound of fusiform cells having spindle shaped ovoid shaped nuclei and irregularly distributed connective tissue fibers. small number of interstitial cells occurs in the ovarian stroma. The interstitial cells are some what larger than the stromal cells, and are polygonal or polyhedral in shape. The interstitial cells are closely packed and in contact with the neighbouring cells which any intervening tissue except for blood capillaries. blood capillaries we re evenly scattered through the gland. The interstitial cells are seen in bunches. The interstitial cells are 3 to 15 in numbers in various different bunches. Blood capillaries are supplied with the interstitial cells in some places

Interstitial cells in inactive ovary of Rousettus leschenaulti

An animal found inactive in August to November. The peripheral part of the ovary contains a large number of primordial follicles, developed into unilaminar, bilaminar, multilaminar follicles, which reach up to a early vesicular stage of follicular development undergo atresia. All the follicles are distributed in stromal area. Made up of ovoid cells and occupy a major part of the ovary.

This animal breeds twice in the year so that pregnant females occur in all the months of the year except August to November. The breast feeding fe male come to heat again and undergo copulation and carry second preganancy. So that the structure of interstitial cells is very much variable in this animal. The number and sizes of interstitial cells are vary. In inactive animal ovary shows $41 \pm 5$ bunches of inters titial cells in the stromal area in between the follicles. These cells are some what bigger to that of stromal cells. In each bundle there are about 5 to 7 interstitial cells measure about 5 to 10 $\square \square \square T h e$ size of interstitial cells in inactive ovary is found to be smaller than that of the active ovary and the interstitial cells is inactive ovary is less hypertrophid than that of active ovary (Fig5a,5b).

\section{Interstitial cells in Active Ovary of Rousettus} leschenaulti

many germinal epithelium cells are enlarged to form the epithelial nodules. Epithelial nodules are the fore runner of the future oocytes. There are not much differences in the structure of the ovarian stroma in the ovaries of sexually inactive and sexually active specimens. In both these cases the stroma is composed of fusiform cells having spindle shaped ovoid nuclei and irregularly dispursed connective tissue fibres.

Interstitial cells are occur in stromal area. These cells are polyhedral and polygonal in shape and larger than the stromal cells. These cells are supplied with blood vessels and capillaries. Interstitial cells are polygonal in shape with spherical nuclei found distributed in stromal region. There are $47 \pm 5$ smaller and larger bunches of interstitial cells are scattered in the pherepheral stromal area to deeper stromal area. There are about 7 to 20 number of interstitial cells in each bunch. interstitial cells are also found in the vicinity of atretic follicles. In the active ovary thecal as well as stromal interstitial cells mixed together and found dominated with stromal area. The size of interstitial cells are measure about 9 to $12 \mu$. The size of interstitial cells in active ovary is larger than that of inactive ovary being the interstitial cells in active ovary is more hypertrophied than the inactive ovary (Fig6).

The pregnant ovary of Rousettus leschenaulti observed degenerating follicles and interstitial cells. One of the function of interstitial cell is to degene rate follicles when function is over (Fig7).

\section{Discussion:-}

As per the above observation it is found that the interstitial cells are found in the ovary of immature and mature animals both. In inactive and active stages of ovaries of Hipposideros speoris and Rousettus leschenaulti bats showed some what structural diffe rences. Some observations resulted that the interstitial cells or Epithelial cords are seen throughout the reproductive cycle in the species Hipposideros speoris. The epithelial cords or interstitial cells originated either by the invagination of the germinal epithelium and from the hypertrophy of persistant granulosa cells of atre tic primodial and preantral follicles (M.S. sasty and S.B. Pillai (2013)

In my observations it is seen that the interstitial cells found in Hipposideros speoris in inactive as well as active ovary, but it is not differentiated from the stromal cells in case of 
immature ovary. In Rousettus leschenaulti, interstitial cells observed in inactive as well as active ovary and it is also diffe re ntiated from the stromal cells, in case of immature ovary. In the immature ovary of Rousettus leschenaulti numerous interstitial cells observed in the stromal area. interstitial cells in ovary numerous in new born bats in myotis lucifugus lucifugus and they are arose from hypertrophied theca internae of retrogressing follicles of granulosa cells. (Guthrie and Jeffer, 1938a). Interstitial cells of thecal origin are in the mammalian ovary (Mossaman et al, 1964). Several type of glandular tissue in the mamalian ovary which are severally described as interstitial cells. Watzka (1957) stated that the primary function of the interstitial cells tis sue was nourishment and destruction of follicles and made no distinction between the thecal gland cells and the interstitial cells.

M.S. Sastry and S.B. Pillai (2013) observed the Hipposiderous speoris ovary and resulted that, the epithelial cords i.e. interstitial cells were observed throughout the reproductive cycle. The epithelial cords have originated either by the invagination of the germinal epithelium and from the hypertrophied of persistant granulosa of atretic primodial and preantral follicles. Further study shows that varying numbers of cords and number of interstitial cells in various developmental stage s.

Antonin Bukvsky et al (2002) studied rat ovarian interstitial cells and described the 5 stages of interstitial cells development.

Immature - small (5 to $7 \mu \mathrm{m}$ ), Younglarge (8 to $10 \mu \mathrm{m})$, Mature - large (12 to $16 \mu \mathrm{m}$ ), Aged - large (12 to $16 \mu \mathrm{m})$, Regressing -Shrink (5 to $7 \mu \mathrm{m}$ ) In the pregnant Rousettus leschenaulti destruction of follicles as well as interstitial cells found.

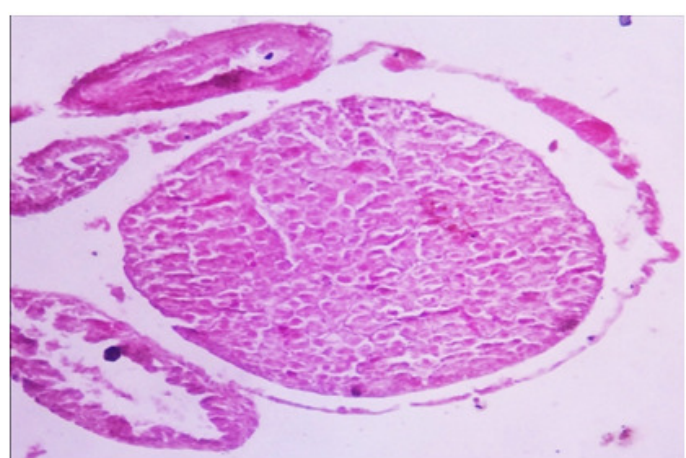

Figure-1a Immature ovary of Hipposiderous speoris showing various developing primordial follicles. Interstitial cells not identified from stro mal cells $\mathrm{x} 100$.

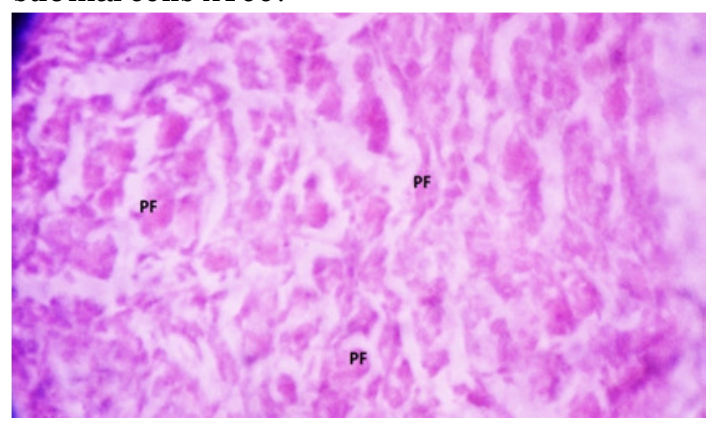

Figure-1b Immature ovary of Hipposiderous speoris showing various developing primordial follicles. Interstitial cells not identified from stro mal cells. X400

$\mathrm{PF}$ - primordial follicles

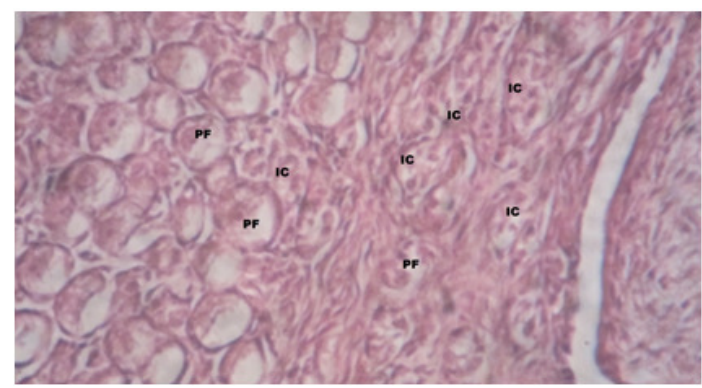

Figure- 2 Inactive ovary of hipposide rous speoris showing large number of primodial follicles and clearly seen interstitial cell bunches distributed all over the stromal area. X400 PF- Primordial Follicles, IC-Interstitial Cells

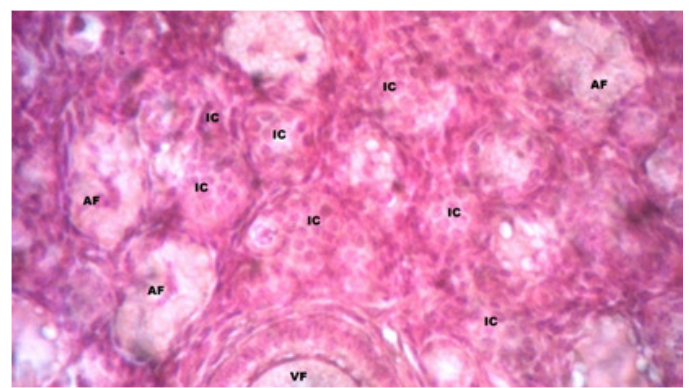

Figure-3 Active ovary of hipposiderous speoris showing follicle and atretic folliclesalongwith nume rous bunches of intestitial cells distributed all over the stromal area.x600

IC- Interstitial Cells, AF- Atretic Follicle, VFVesicular Follicle 


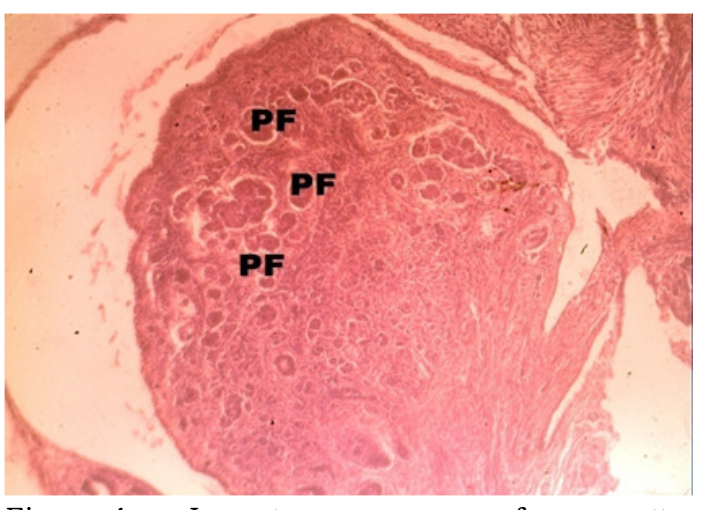

Figure-4a Immature ovary of rousettus lechenaulti showing deve loping follicles $\mathrm{x} 100$ PF- Primordial Follicles

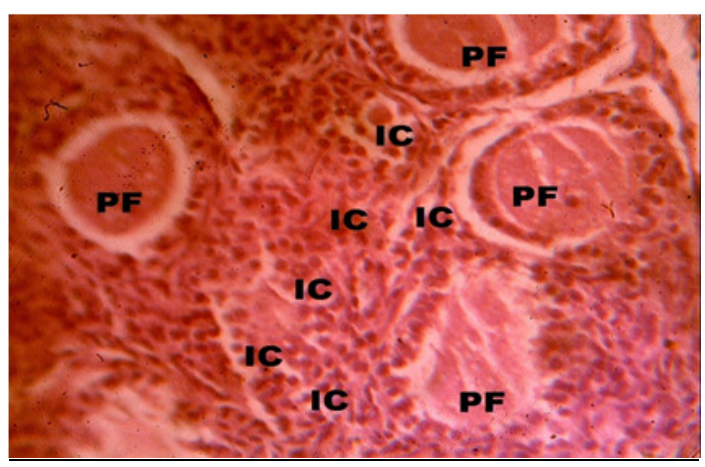

Figure $4 \mathrm{~b}$ Immature ovary of Rousettus leschenaulti showing primordial follicles and also clearly seen interstitial cell bunches in large stromal area. X400

PF- Primordial Follicles, IC- Inters titial Cells

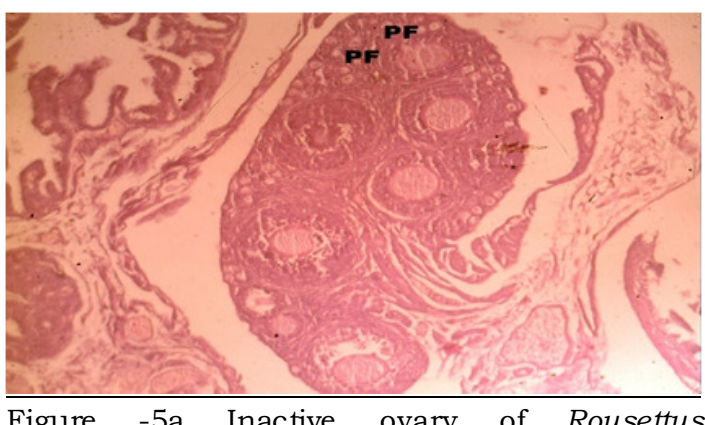

leschenaulti showing various developing follicles and large stromal area with interstitial cell bunches. 4400

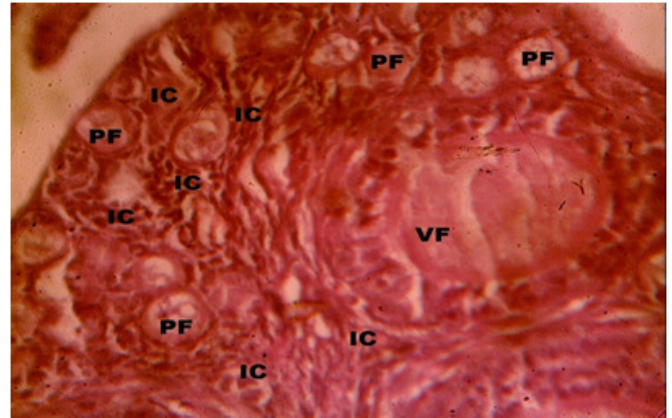

Figure- 5b Inactive ovary of Rousettus leschenaulti showing two laye red follicle, primordial follicle and interstitial cell bunches in stromal are a in the periphery. X400

$\mathrm{PF}$ - Primordial Follicle s IC- Interstitial Cells, VFVesicular Follicle

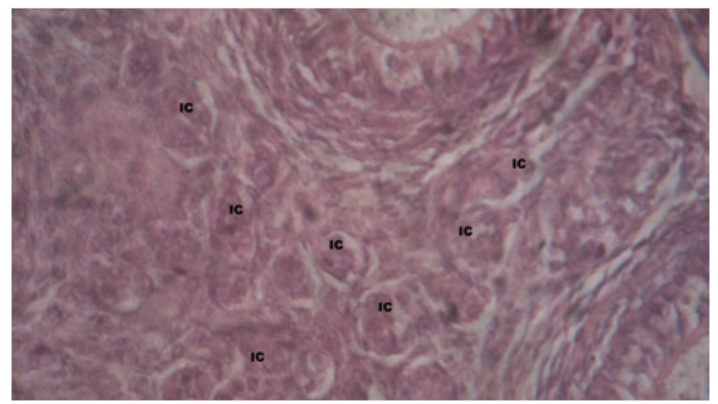

Figure -6 Active ovary of Rousettus leschenaulti showing interstitial cell bunches in stromal are a along with 3-4 layered follicles. X400 IC- Inte rs titial Cells

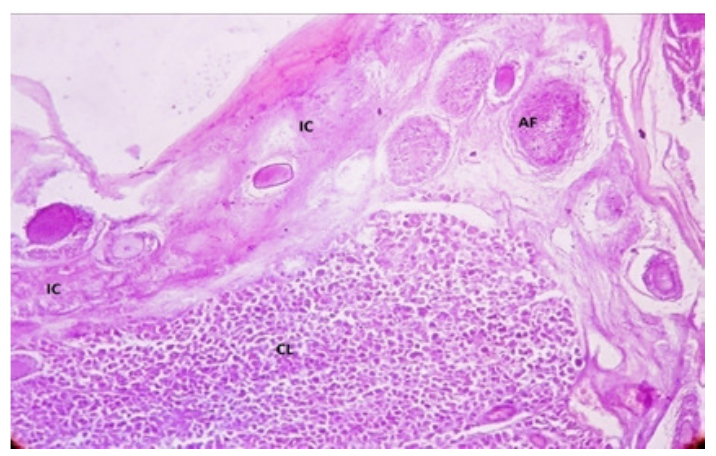

Figure $\quad-7$ Pregnant ovary of Rousettus leschenaulti showing destroying follicles and disintigrated interstitial cells alongwith major part of introverted carpus leu teum x 100 .

AF- Atretic Follicle, IC- Interstitial Cells, CLCarpus Leuteum 


\section{References}

Brambell FWR (1966) ovarian changes in Marshall's physiology of Reproduction" (As parks ed.) Vol. III, pp. 397-542, Little Brown and Co. Boston.

Guraya SS (1967), Cytochemincal study of interstitial cells in the bat ovary. Natyze, 214 : 614-616

Guraya SS and Green wolds GS (1964) A comparative histochimical study of interstitial tissue and follicular atresia in the mammalian ovary. The Anatomical Record, Vol 149, is sue 3, 411-433

Guthrie MJ and Jeffers KRC (1938a) A Cytoclogical studies of ovaries of the bats, relyotis grisen cens and myotis lunifugus lucifugus. J Morph, 65.523-557

M.S. Sastry and Dharma Tembhare (2009) studied the ovaries of Hipposiderous speoris and observed that the stromal type of interstitial gland cells in much of the cortex and medulla in both the functional and non-functional ovaries.

Mossman H W and Koering MJ (1964) Cyclie changes of interstitial gland tissue of the human ovary. American Journal of Anatomy, Vol. 115, Issue 2, page 235-255

Sastry MS and Pillai S B (2013) Variations in the epithelis cords of the ovaries of a microchiropteran bat, Hipposiderous speoris (schneider) during reproductive cycle : An enzymic approach journal of cell and Animal Biology. Vol 7 (II) : 132-137.

Watzka M (1951) weiblicbe Genitablorgane. Das ovarium In handbuch Mika. Anat Menschen III 178 pp. (Ed. W Von Mollendorff and W Bargmann) Berlin : Springer. 